\title{
Returning pharmacogenetic secondary findings from genome sequencing: let's not put the cart before the horse
}

\author{
A. Cecile J.W. Janssens, $\mathrm{PhD}^{1,2}$ and James P. Evans, MD, $\mathrm{PhD}^{3}$
}

Whole-genome and whole-exome sequencing are taking off in health care. DNA sequencing was introduced to enable the molecular diagnosis of congenital disorders and rare syndromes, followed by several studies that explored the introduction of sequencing in various clinical settings. ${ }^{1,2}$ Given the role of DNA in many diseases and outcomes, it is understandable that there is research interest in the secondary use of sequencing data and in the preemptive sequencing of healthy individuals in the event that DNA data are needed for a later medical decision.

Expectations regarding the impact of DNA sequencing in health-care practice are high. Many researchers have already investigated the expectations, views, and attitudes toward receiving (secondary) findings from sequencing among African Americans $^{3}$ and non-African Americans ${ }^{4}$; among genetic, ${ }^{5}$ pediatric, ${ }^{6}$ primary care, ${ }^{7}$ and nonmedical health professionals $^{8}$; and about sequencing in patients with Lynch syndrome ${ }^{9}$ as well as newborns ${ }^{10}$ and children. ${ }^{11}$ Several studies went beyond views and addressed intentions, ${ }^{12}$ knowledge, awareness, and understanding ${ }^{13}$; whereas others focused on the practical aspects of how to integrate sequencing in health-care practice ${ }^{14}$; how to deliver the service ${ }^{15}$; how to prepare different health professionals such as nurses ${ }^{16}$ and genetic counselors ${ }^{17}$ for their role in the delivery; how to design the patient report ${ }^{18}$; and how to handle informed consent in adults, ${ }^{19}$ children, ${ }^{20}$ and in families with genetic disease. ${ }^{21}$ We already have a glimpse of the usefulness ${ }^{22}$ and cost-effectiveness of returning secondary findings. ${ }^{23}$ All in one year.

The study by Nishimura and colleagues ${ }^{24}$ in this issue of Genetics in Medicine is similar. It addresses pharmacogenetics (PGx)-perhaps the single category of secondary results that has been most trumpeted for potential clinical utility. Nishimura and colleagues present proof of concept on how alerts for secondary PGx findings from genome sequencing can be automated in electronic health records. According to the authors, "incidental findings can be used to generate decision support alerts, [but] substantial resources are required to ensure that each alert is consistent with rapidly evolving pharmacogenomics literature and is customized to fit in the clinical workflow unique to each incidental finding." Stated very simply, and slightly skeptically, their study showed that alerts for secondary PGx findings can be programmed but that we do not really know what to program.

The authors' conclusion reveals that there were two challenges in their study: can we technically program alerts for the return of secondary pharmacogenetic findings into electronic health records, and do we know how to identify those findings that are worth returning? The first question is not a scientific question but a technological one, and it probably has a very simple answer: yes, we can. If we can program bionic eyes and driverless cars, it seems highly likely that we can program straightforward, but likely context-dependent, alerts into an electronic health record system. The authors were able to produce alerts, 49 in 54 patients, but they did not comment on whether these alerts were correct (did the alert return the right information?), relevant (was the patient described drugs related to the reported pharmacogenetic association?), and useful (would such a change actually benefit patients?).

The second question is the real challenge: do we know what is worth returning? Well, not really. There are a handful of pharmacogenetic associations that affect drug response or safety beyond doubt, such as HLA for abacavir and CYP2D6 for codeine, ${ }^{25,26}$ but for most others there is at least substantial doubt about the utility of testing and even the robustness of the pharmacogenetic association. For the list tested by Nishimura and colleagues, ${ }^{24} 7$ of 11 variants (Table 1 in their article) are recommended by the Clinical Pharmacogenetics Implementation Consortium, of which only two also are recommended (but not required) by the US Food and Drug Administration. ${ }^{27}$

The authors are well aware of the ambiguity about what to return and argue that "substantial resources are required to ensure that each alert is consistent with rapidly evolving pharmacogenomics literature." The ambiguity equates a fundamental scientific question: when do we have enough evidence that we can conclude a pharmacogenetic association is true? And is it true that the evidence is changing rapidly?

Science is a process in which evidence typically accumulates through the synthesis of individual studies. In the classical approach these studies aim to falsify a hypothesis by attempting to demonstrate that it is not true. For example, the

\footnotetext{
${ }^{1}$ Department of Epidemiology, Rollins School of Public Health, Emory University, Atlanta, Georgia, USA; ${ }^{2}$ Department of Clinical Genetics/EMGO Institute for Health and Care Research, Section of Community Genetics, VU University Medical Center, Amsterdam, The Netherlands; ${ }^{3}$ Department of Genetics, University of North Carolina at Chapel Hill, Chapel Hill, North Carolina, USA. Correspondence: A. Cecile J. W. Janssens (cecile.janssens@emory.edu) 
null hypothesis specifies that there is no association between a genetic variant and drug response. Every valid attempt in a methodologically sound study with sufficient statistical power that would fail to show no effect, and hence reject the null hypothesis, would make it more likely that the alternate hypothesis (i.e., that there is an association) is true. While the existence of an association can never be proven with certainty, at some point the accumulation of evidence can be evaluated as strong enough to conclude the presence of association.

A more fundamental point, however, is that even if a statistical correlation exists, say between a PGx variant and drug levels, this remains a far cry from establishing that such an association has any clinical relevance. Indeed, given the number of factors that influence drug efficacy and adverse reactions, any given PGx variant, regardless of the existence of a statistical correlation, will not necessarily matter in any meaningful way from a patient's perspective. This is illustrated robustly by recent work demonstrating that PGx guidance for warfarin dosing, long a poster child for the PGx field, simply does not matter when using this agent. ${ }^{28,29}$

Thus, while many associations are suggested by ongoing studies, not all remain statistically significant after replication studies, and few reach a level at which clinical implementation is warranted. Thus, the notion, suggested by Nishimura et al., ${ }^{24}$ that substantial resources are required to keep their alerts up to date is questionable. In reality, those resources would be far better used to determine what PGx associations (probably few, given the track record thus far) may actually improve patient outcomes. The responsible approach to the introduction of pharmacogenetic testing, or to the use of genetic data when collected preemptively, is to wait until the scientific evidence is robust enough to warrant its use. This advice holds as well for studies that examine people's attitudes, preferences, and intentions; how to design bioinformatics pipelines; and the design of counseling services and informed consent. Such studies will be needed but are meaningful only when it is clear what genetic tests should ultimately be introduced in practice.

Finally, we should also not be seduced by the facile notion that if one engages in whole-exome sequencing and generates secondary information (such as PGx data), that such information is "free" and now ripe for clinical application. Whether clinical information is initially generated at high cost, low cost, or no cost is beside the point. The clinical use of information that does not improve patient outcomes just because it is "free" ignores the fact that the inappropriate use of medical data has substantial downstream costs in terms of both patient wellbeing and money. Moreover, opportunity costs are very real in medicine; the inappropriate use of data to guide clinical decisions inevitably precludes more important expenditures of time and resources in patient care.

It is understandable that the potential of PGx to improve patient care has generated excitement. The notion that we could guide the use of drugs through genomic information is tantalizing. But unfortunately, that vision remains far from realized for the vast majority of medications after enormous expenditures of time and money. Given that after many years of effort very few examples of PGx application have shown much promise for actually improving patient outcomes, we should recognize the likely reality that the role of PGx guidance will be limited to relatively few agents.

We do not propose to thwart scientific progress, but, rather, to simply advocate for spending precious time and resources wisely. The appropriate first steps before introducing preemptive sequencing into the routine clinical arena is to demonstrate clinical validity and at least a reasonable suggestion of clinical utility. ${ }^{30}$ So far, for the vast majority of pharmacogenetic tests, the evidence lags far behind.

\section{DISCLOSURE}

The authors declare no conflict of interest.

\section{REFERENCES}

1. Vassy JL, Lautenbach DM, McLaughlin HM, et al.; MedSeq Project. The MedSeq Project: a randomized trial of integrating whole genome sequencing into clinical medicine. Trials 2014;15:85.

2. Biesecker LG, Mullikin JC, Facio FM, et al.; NISC Comparative Sequencing Program. The ClinSeq Project: piloting large-scale genome sequencing for research in genomic medicine. Genome Res 2009;19:1665-1674.

3. Yu JH, Crouch J, Jamal SM, Tabor HK, Bamshad MJ. Attitudes of African Americans toward return of results from exome and whole genome sequencing. Am J Med Genet A 2013;161A:1064-1072.

4. Yu JH, Crouch J, Jamal SM, Bamshad MJ, Tabor HK. Attitudes of non-African American focus group participants toward return of results from exome and whole genome sequencing. Am J Med Genet A 2014;164A:2153-2160.

5. Yu JH, Harrell TM, Jamal SM, Tabor HK, Bamshad MJ. Attitudes of genetics professionals toward the return of incidental results from exome and wholegenome sequencing. Am J Hum Genet 2014;95:77-84.

6. Barajas M, Ross LF. Pediatric professionals' attitudes about secondary findings in genomic sequencing of children. J Pediatr; e-pub ahead of print 26 February 2015.

7. Strong KA, Zusevics KL, Bick D, Veith R. Views of primary care providers regarding the return of genome sequencing incidental findings. Clin Genet 2014;86:461-468.

8. Strong A, Zusevics L, Bick DP, Veith R. Views of nonmedical, health system professionals regarding the return of whole genome sequencing incidental findings. WMJ 2014;113:179-184.

9. Hitch K, Joseph G, Guiltinan J, Kianmahd J, Youngblom J, Blanco A. Lynch syndrome patients' views of and preferences for return of results following whole exome sequencing. J Genet Couns 2014;23:539-551.

10. Goldenberg AJ, Dodson DS, Davis MM, Tarini BA. Parents' interest in wholegenome sequencing of newborns. Genet Med 2014;16:78-84.

11. Sapp JC, Dong D, Stark C, et al. Parental attitudes, values, and beliefs toward the return of results from exome sequencing in children. Clin Genet 2014;85:120-126.

12. Facio FM, Eidem $\mathrm{H}$, Fisher $\mathrm{T}$, et al. Intentions to receive individual results from whole-genome sequencing among participants in the ClinSeq study. Eur J Hum Genet 2013;21:261-265.

13. Boland PM, Ruth K, Matro JM, et al. Genetic counselors' (GC) knowledge, awareness, understanding of clinical next-generation sequencing (NGS) genomic testing. Clin Genet; e-pub ahead of print 19 December 2014.

14. Mauer CB, Pirzadeh-Miller SM, Robinson LD, Euhus DM. The integration of next-generation sequencing panels in the clinical cancer genetics practice: an institutional experience. Genet Med 2014;16:407-412.

15. Wright MF, Lewis KL, Fisher TC, et al. Preferences for results delivery from exome sequencing/genome sequencing. Genet Med 2014;16:442-447.

16. Prows CA, Tran G, Blosser B. Whole exome or genome sequencing: nurses need to prepare families for the possibilities. J Adv Nurs 2014;70:2736-2745.

17. Wolfe Schneider K, Anguiano A, Axell L, et al. Collaboration of colorado cancer genetic counselors to integrate next generation sequencing panels into clinical practice. J Genet Couns 2014;23:640-646.

18. McLaughlin HM, Ceyhan-Birsoy O, Christensen KD, et al.; MedSeq Project. A systematic approach to the reporting of medically relevant findings from whole genome sequencing. BMC Med Genet 2014;15:134. 
19. Ayuso C, Millán JM, Mancheño M, Dal-Ré R. Informed consent for wholegenome sequencing studies in the clinical setting. Proposed recommendations on essential content and process. Eur J Hum Genet 2013;21:1054-1059.

20. Levenseller BL, Soucier DJ, Miller VA, Harris D, Conway L, Bernhardt BA. Stakeholders' opinions on the implementation of pediatric whole exome sequencing: implications for informed consent. J Genet Couns 2014;23:552-565.

21. Bergner AL, Bollinger J, Raraigh $\mathrm{KS}$, et al. Informed consent for exome sequencing research in families with genetic disease: the emerging issue of incidental findings. Am J Med Genet A 2014;164A:2745-2752.

22. Iglesias A, Anyane-Yeboa K, Wynn J, et al. The usefulness of whole-exome sequencing in routine clinical practice. Genet Med 2014;16:922-931.

23. Bennette CS, Gallego CJ, Burke W, et al. The cost-effectiveness of returning incidental findings from next-generation genomic sequencing. Genet Med; e-pub ahead of print 13 November 2014.

24. Nishimura AA, Shirts BH, Dorschner MO, et al. Development of clinical decision support alerts for pharmacogenomic incidental findings from exome sequencing. Genet Med; epub ahead of print 5 March, 2015.
25. Mallal S, Phillips E, Carosi G, et al.; PREDICT-1 Study Team. HLA$B * 5701$ screening for hypersensitivity to abacavir. N Engl J Med 2008;358: 568-579.

26. Nicholson WT, Formea CM. Clinical perspective on the Clinical Pharmacogenetics Implementation Consortium Updated 2014 guidelines for CYP2D6 and codeine. Clin Chem 2015;61:319-321.

27. PharmGKB. Secondary PharmGKB 2015. http://www.pharmgkb.org/cpic/ pairs.

28. Kimmel SE, French B, Kasner SE, et al.; COAG Investigators. A pharmacogenetic versus a clinical algorithm for warfarin dosing. N Engl J Med 2013;369: 2283-2293.

29. Pirmohamed M, Burnside G, Eriksson N, et al.; EU-PACT Group. A randomized trial of genotype-guided dosing of warfarin. N Engl J Med 2013;369: 2294-2303.

30. Janssens AC, Deverka PA. Useless until proven effective: the clinical utility of preemptive pharmacogenetic testing. Clin Pharmacol Ther 2014;96: 652-654. 